\title{
Breast Surgery in 2015: Advances in Recent Years
}

\author{
Judy C. Boughey, $\mathrm{MD}^{1}$ and Sarah A. McLaughlin, $\mathrm{MD}^{2}$ \\ ${ }^{1}$ Mayo Clinic, Rochester, MN; ${ }^{2}$ Mayo Clinic, Jacksonville, FL
}

Welcome to the October 2015 issue of the Annals of Surgical Oncology, dedicated to the annual meeting of the American Society of Breast Surgeons (ASBrS). The 16th annual meeting was held at Grande Lakes Orlando, FL, April 29 to May 3, 2015. The society and its annual meeting continue to grow year to year, and once again it was a highly successful meeting, with over 1400 attendees. New at the meeting this year was the first American Society of Breast Surgeons/Arnold P. Gold Foundation Humanism and Medicine Award, which was awarded to Brian Czerniecki, MD, PhD. Dr. Czerniecki is RhodesHarrington Professor of Surgical Oncology at the University of Pennsylvania and the codirector of the Rena Rowan Breast Center. His research interests focus on dendritic cell biology and interactions with $\mathrm{T}$ cells. He has developed dendritic cell vaccines for the treatment of cancer and is involved with several clinical trials for treating patients with early breast cancer with dendritic cell vaccines.

In this issue we include the provoking presidential address from Dr. Hiram Cody from Memorial Sloan Kettering Cancer Center and also the keynote lecture delivered by Dr. Monica Morrow on rethinking the local therapy of breast cancer-integration of biology and anatomy. ${ }^{1,2}$ In addition, we include invited articles from selected sessions from the annual meeting, as well as scientific publications from the oral session, quick-shots session, and poster session.

The meeting, as always, provided up-to-date information on the current recommendations for management of patients with benign breast disease and with breast malignancies. It also provided us with the opportunity to look back at the changes and the advances in the surgical

(C) Society of Surgical Oncology 2015

First Received: 30 May 2015;

Published Online: 5 August 2015

J. C. Boughey, MD

e-mail: boughey.judy@mayo.edu management of breast disease over the last 10 years. In addition to the usual best papers of the past year, Dr. Helen Pass provided an overview of the most practice changing publications over the last 10 years. This gave us all opportunity to reflect on how far our field has advanced in such a short amount of time. For this year's editorial, we have chosen to highlight five key areas of breast surgery where significant changes or advances have been made in the last 10 years.

\section{NIPPLE-SPARING MASTECTOMY (NSM)}

NSM has become much more commonly performed over the last 10 years than ever before. Initially, in the 1960s, NSM was synonymous with subcutaneous mastectomy, was frequently performed by plastic surgeons, and was used primarily in the setting of risk reduction. Now, in 2015 , NSM is commonly utilized both for prophylactic surgery and for therapeutic purposes in selected patients with breast cancer. Although the long-term follow-up of NSM remains more limited than our experience with simple mastectomy or skin-sparing mastectomy, the data are continually accumulating. A meta-analysis of published outcome data of NSM in relation to local recurrence in the nipple-areolar complex, as well as disease-free survival and overall survival, was presented at this year's meeting; the article is included in this issue. ${ }^{3}$ The data presented are reassuring and show low nipple areolar recurrence rates and similar overall survival and disease-free survival to other mastectomy groups. In fact, the better survival data in the NSM group likely reflect a better overall patient group in terms of tumor and/or patient comorbidities, which were not able to be completely controlled for in this metaanalysis. It remains important to realize that the majority of patients selected for NSM, and included in the publications included in this meta-analysis, were likely well selected by their surgeons. Most patients with NSM that we have longer follow-up on are the patients from earlier in our 
experience, when we were more conservative in patient selection. Over time, indications for NSM have expanded to larger tumors, larger breasts, and shorter distance of disease from nipple. ${ }^{4}$ Thus, the currently available outcome data may not necessarily be ideal to extrapolate to those patients (such as those with larger tumors) where previously we may have not considered NSM.

In appropriately selected patients, NSM provides good oncologic control along with superior cosmetic outcome. This has allowed us to move away from mastectomy being a disfiguring operation and to provide appropriate patients with an option that preserves their breast skin and nipple areola. Current surgical techniques with lateral or inframammary skin incisions and reconstruction techniques allowing for immediate implant or nearcomplete tissue expansion have facilitated treatment and recovery with minimal psychological impact from loss of a breast mound or nipple and provide excellent cosmetic results. ${ }^{5}$

\section{CONTRALATERAL PROPHYLACTIC MASTECTOMY (CPM)}

For women diagnosed with a unilateral breast cancer without a strong family history or a known $B R C A$ mutation, the risk of a contralateral breast malignancy has been decreasing over time. ${ }^{6,7}$ Most women with hormone receptor-positive disease receive endocrine therapy, which further protects the contralateral breast by halving the risk of contralateral malignancy. Thus, the overall risk benefit has failed to show significant survival advantage for CPM in most women with unilateral breast cancer. Those patients who may derive some survival benefit from CPM are younger women with triple-negative breast cancer. ${ }^{8,9}$ However, despite these data, the national rates of CPM continue to increase dramatically. This has been a point of significant debate in the media as well as at national breast meetings over the past several years. ${ }^{10,11}$ This year's ASBrS meeting had an excellent session that included discussions of the risks, ethics, and outcomes of CPM. As our techniques for mastectomy, such as NSM, and reconstruction, such as immediate implant or autologous-based breast reconstruction, improve, there seems to be an unanticipated effect of increasing rates of CPM. Where previously simple mastectomy without reconstruction was once a procedure that patients considered disfiguring, the balance has changed, and symmetry is now a common concern of patients for consideration after oncologic factors. Patient-reported outcome data show that patients undergoing unilateral mastectomy with implant-based reconstruction have the lowest satisfaction scores, thus suggesting cosmetic reasons to be a driver for CPM. ${ }^{12-14}$

\section{AXILLARY SURGERY}

Interestingly, as management of the breast has trended to be more extensive, with an increase in mastectomy rather than an increase in breast conservation, management of the axilla has succeeded in becoming less extensive. This is multifactorial and is due to advances in radiology as well as surgery, systemic therapy, and radiotherapy. The changes in management of the axilla span from early-stage breast cancer to advanced disease. Routine use of axillary ultrasound for patients with invasive breast cancer has improved clinical staging and allowed identification of node-positive disease before surgical resection in many cases. ${ }^{15}$ This informs patients of their nodal status and aids in discussions about neoadjuvant systemic therapy as well as the surgical approach to the axilla. The Z0011 trial results have significantly changed the surgical management of the axilla, supporting the omission of axillary node dissection when one or two positive nodes are found in patients with clinical T1-2 N0 disease who complete breast conservation surgery and are planning whole-breast radiotherapy. ${ }^{16}$ This has resulted in less axillary dissections done nationally. One could hypothesize that the opportunity for axillary conservation for patients pursuing breast conservation may ultimately lead to an increase in breast conservation rates because node-positive patients pursuing mastectomy are still recommended axillary dissection.

At the other end of the spectrum are those patients treated with neoadjuvant chemotherapy. Whereas previously in cases of documented node-positive disease before chemotherapy the surgical recommendation after chemotherapy was axillary lymph node dissection, the striking improvements in pathologic complete response rates seen with the use of targeted agents in the neoadjuvant setting now suggest that the maximally invasive approach of axillary dissection for all patients may not be necessary. ACOSOG Z1071, the SENTINA trial in Europe, and the Canadian SN FNAC study showed that the falsenegative rate of sentinel lymph node surgery after chemotherapy for patients presenting with node-positive breast cancer was $8-14 \% .{ }^{17-19}$ Factors that decreased the false-negative rate was the resection of two or more sentinel lymph nodes, the use of dual-agent tracers, the use of immunohistochemistry, and the placement of a clip in the positive node at diagnosis with removal of the node containing that clip at surgery..$^{17,18,20}$ The National Comprehensive Cancer Network guidelines now list sentinel lymph node surgery as an option in these cases. Thus, increasingly across the country, patients with node-positive breast cancer treated with neoadjuvant chemotherapy can undergo sentinel lymph node surgery to evaluate the nodal response to chemotherapy and reserve axillary dissection for residual nodal disease. 


\section{MARGINS FOR BREAST-CONSERVING SURGERY}

Despite conclusive evidence from mature randomized controlled trials demonstrating equivalence of breastconservation therapy (lumpectomy and whole breast irradiation) and mastectomy, what constitutes a negative margin after breast-conserving surgery has long remained a controversial topic. Not only have surgeons disagreed on the width of a negative margin but researchers have also documented substantial variability in reoperation rates. ${ }^{21}$ This past year has witnessed significant progress to solving these issues with publication and widespread endorsement of the SSO-ASTRO margin consensus statement. ${ }^{22}$ Championed by Drs. Monica Morrow (SSO) and Meena Moran (ASTRO), this multidisciplinary panel performed a systematic review and meta-analysis of 33 studies and over 28,000 patients. This consensus statement clearly states the use of no ink on tumor as an adequate margin in lumpectomy for invasive cancer in the era of multidisciplinary treatment. Dr. Morrow reviewed these data extensively in her keynote address (published in this issue), noting that rates of in-breast tumor recurrence are influenced by biologic subtype and effectively reduced by systemic therapy and radiotherapy. ${ }^{2}$ Even in young patients $<40$ years old, those with invasive lobular cancer, or those with tumors with extensive intraductal component, no evidence exists supporting a wider margin to further reduce in-breast tumor recurrence risk. The intended consequences of this consensus statement are to reduce reoperation rates and thereby improve cosmesis as well as decrease treatment costs. An oral presentation at this year's meetings highlighted the rapid adoption of the consensus statement into clinical practice, noting that $95 \%$ of ASBrS surgeons agree with the definition of no ink on tumor and suggesting clear trends toward reduced reexcisions. ${ }^{23}$ Importantly, they also suggest that surgeons are continuing to use their judgement in the application of the guidelines, as stressed by ASCO in situations of clinical discordance, extensive microcalcifications, and multidisciplinary discussion. ${ }^{24,25}$

\section{GENETIC TESTING}

In keeping with the theme of personalizing therapy, multiple components of this year's annual meeting focused on the challenges and controversies surrounding genetic testing. With the Supreme Court's decision against Myriad Genetics, citing the illegality of patenting genes, clinicians have experienced an upsurge in the commercialization and widespread availability of a variety of different genetic tests. The guidelines encompassing who should be tested have been expanded and now include not only those women with suspicious personal or family histories of breast or ovarian cancers but also include all women with triple-negative breast cancer diagnosed before age 60, premenopausal women (age $<50$ ) with breast cancer, and any patients with a personal or family history of more than three specific cancers (pancreas, prostate, sarcoma, adrenocortical, gastric, brain, endometrial, thyroid, renal). ${ }^{26}$ Further, available multigene panels now have the capability of testing for many more genes than just BRCAl/ 2. Although these can be informative of additional highrisk mutations, they are also associated with the increased likelihood of finding variants of unknown significance. Therefore, this explosion in genetic testing has implications beyond ordering the right test, and it mandates the appropriate counseling of patients. Specifically, it highlights the key symbiotic relationship that must exist between surgeons and genetic counselors and provides a unique opportunity for our two societies to work collectively to solve what is undoubtedly a national issue. The article in this issue by Dr. Pat Whitworth and Joy Larsen Haidle, MS, CGC president of the National Society of Genetic Counselors, further explores these challenges. ${ }^{27}$ Finally, as more patients undergo genetic testing, surgeons will be faced with mutations in rare or unknown genes. Vast gene libraries and comprehensive family pedigrees will be critical to determining the meaning of these genes, as Dr. David Euhus nicely outlined during the CPM session. ${ }^{28}$ Clearly, surgeons' education in breast care in 2015 must extend beyond technical skills.

In conclusion, although the cornerstones of breast cancer care, including breast-conservation therapy, mastectomy, and axillary evaluation, have remained unchanged, it is clear that optimizing and personalizing treatment choices for our patients is crucial. We continue to challenge existing dogma in an effort to do less, but to do it with oncologic safety and with the needs of the patient as the highest priority. Surgeons have long been ahead of the curve in adopting progressive change for breast cancer care. There is no doubt in this era of improved understanding of tumor biology, targeted systemic therapy, and genomics that further multidisciplinary individualization of treatment strategies will ensue.

DISCLOSURE The authors declare no conflict of interest.

\section{REFERENCES}

1. Cody HS III. Presidential address: "A part of the main". Ann Surg Oncol. 2015. doi:10.1245/s10434-015-4667-0.

2. Morrow M. Rethinking the local therapy of breast cancer: integration of biology and anatomy. Ann Surg Oncology. 2015. doi:10.1245/s10434-015-4750-6.

3. De la Cruz L, Moody AM, Tappy EE, et al. Overall survival, disease-free survival, local recurrence, and nipple-areolar recurrence in the setting of nipple-sparing mastectomy: a meta- 
analysis and systematic review. Ann Surg Oncol. 2015. doi:10. 1245/s10434-015-4739-1.

4. Coopey SB, Tang R, Lei L, et al. Increasing eligibility for nipplesparing mastectomy. Ann Surg Oncol. 2013;20:3218-22.

5. Laronga $\mathrm{C}$, Smith $\mathrm{P}$. Nipple-sparing mastectomy: an oncologic and cosmetic perspective. Surg Oncol Clin $N$ Am. 2014;23:549-66.

6. Nichols HB, Berrington de González A, Lacey JV Jr, Rosenberg PS, Anderson WF. Declining incidence of contralateral breast cancer in the United States from 1975 to 2006. J Clin Oncol. 2011;29:1564-9.

7. Early Breast Cancer Trialists' Collaborative Group (EBCTCG); Davies C, Godwin J, et al. Relevance of breast cancer hormone receptors and other factors to the efficacy of adjuvant tamoxifen: patient-level meta-analysis of randomised trials. Lancet. 2011;378(9793):771-84.

8. Bedrosian I, Hu CY, Chang GJ. Population-based study of contralateral prophylactic mastectomy and survival outcomes of breast cancer patients. J Natl Cancer Inst. 2010;102:401-9.

9. Parker PA, Peterson SK, Bedrosian I, et al. Prospective study of surgical decision-making processes for contralateral prophylactic mastectomy in women with breast cancer. Ann Surg. 2015. In press.

10. King TA, Sakr R, Patil S, et al. Clinical management factors contribute to the decision for contralateral prophylactic mastectomy. J Clin Oncol. 2011;29:2158-64.

11. Tuttle TM, Habermann EB, Grund EH, Morris TJ, Virnig BA. Increasing use of contralateral prophylactic mastectomy for breast cancer patients: a trend toward more aggressive surgical treatment. J Clin Oncol. 2007;25:5203-9.

12. Koslow S, Pharmer LA, Scott AM, et al. Long-term patient-reported satisfaction after contralateral prophylactic mastectomy and implant reconstruction. Ann Surg Oncol. 2013;20:3422-9.

13. Scott AM, et al. Patient-reported satisfaction and health relatedquality of life in patients converting from prosthetic to autologous breast reconstruction. Plastic Reconstr Surg. 2014;134(4 suppl 1):89.

14. Atisha DM, Rushing CN, Samsa GP, et al. A national snapshot of satisfaction with breast cancer procedures. Ann Surg Oncol. 2015;22:361-9.

15. Hieken TJ, Trull BC, Boughey JC, et al. Preoperative axillary imaging with percutaneous lymph node biopsy is valuable in the contemporary management of patients with breast cancer. Surgery. 2013;154:831-8

16. Giuliano AE, Hunt KK, Ballman KV, et al. Axillary dissection vs no axillary dissection in women with invasive breast cancer and sentinel node metastasis: a randomized clinical trial. JAMA. 2011;305:569-75.
17. Boughey JC, Suman VJ, Mittendorf EA, et al. Sentinel lymph node surgery after neoadjuvant chemotherapy in patients with node-positive breast cancer: the ACOSOG Z1071 (Alliance) clinical trial. JAMA. 2013;310:1455-61.

18. Kuehn T, Bauerfeind I, Fehm T, et al. Sentinel-lymph-node biopsy in patients with breast cancer before and after neoadjuvant chemotherapy (SENTINA): a prospective, multicentre cohort study. Lancet Oncol. 2013;14:609-18.

19. Boileau JF, Poirier B, Basik M, et al. Sentinel node biopsy after neoadjuvant chemotherapy in biopsy-proven node-positive breast cancer: the SN FNAC study. J Clin Oncol. 2015;33:258-64.

20. Boughey JC, Ballman KV, Le-Petross HT, et al. Identification and resection of the clipped node decreases the false negative rate of sentinel lymph node surgery in patients presenting with node positive breast cancer (T0-T4, N1-2) who receive neoadjuvant chemotherapy-results from ACOSOG Z1071 (Alliance). Ann Surg. In press.

21. McCahill LE, Single RM, Aiello Bowles EJ, et al. Variability in reexcision following breast conservation surgery. JAMA. 2012;307:467-75.

22. Moran MS, Schnitt SJ, Giuliano AE, et al. Society of Surgical Oncology-American Society for Radiation Oncology consensus guideline on margins for breast-conserving surgery with wholebreast irradiation in stages I and II invasive breast cancer. Ann Surg Oncol. 2014;21:704-16.

23. DeSnyder SM, Hunt KK, Smith BD, et al. Assessment of practice patterns following publication of the SSO-ASTRO consensus guideline on margins for breast-conserving therapy in stage I and II invasive breast cancer. Ann Surg Oncol. 2015. doi:10.1245/ s10434-015-4666-1.

24. Buchholz TA, Somerfield MR, Griggs JJ, et al. Margins for breast-conserving surgery with whole-breast irradiation in stage I and II invasive breast cancer: American Society of Clinical Oncology endorsement of the Society of Surgical Oncology/ American Society for Radiation Oncology consensus guideline. $J$ Clin Oncol. 2014;32:1502-6.

25. Harness JK, Giuliano AE, Pockaj BA, Downs-Kelly E. Margins: a status report from the Annual Meeting of the American Society of Breast Surgeons. Ann Surg Oncol. 2014;21:3192-7.

26. NCCN Guidelines Version 1. Genetic/familial high-risk assessment: breast and ovarian. 2015.

27. Haidle JL, Whitworth P. Contemporary challenges in genetic testing for breast cancer: a collaboration opportunity for genetic counselors and breast surgeons. Ann Surg Oncol. 2015. doi:10. 1245/s10434-015-4757-z.

28. Angelos P, Bedrosian I, Euhus DM, et al. Contralateral prophylactic mastectomy: challenging considerations for the surgeon. Ann Surg Oncol. 2015. doi:10.1245/s10434-015-4758-y. 